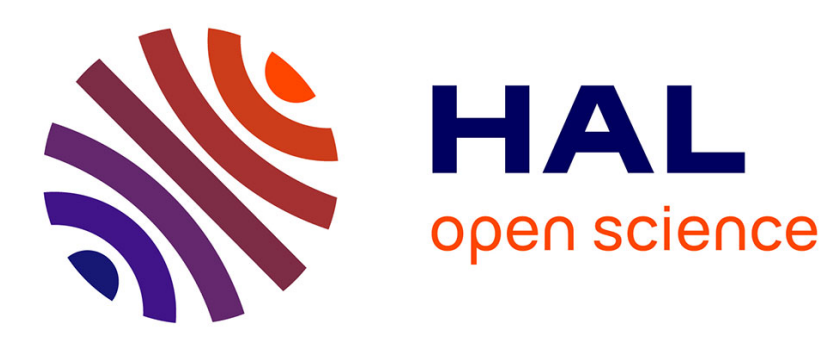

\title{
Sémantique cognitive de l'action : 2 . étude expérimentale de la catégorisation des verbes d'action
}

Christiane Kekenbosch, Jean-Marc Meunier, Jean-Francois Richard, Jean-Pierre Desclés, Valérie Flageul

\section{To cite this version:}

Christiane Kekenbosch, Jean-Marc Meunier, Jean-Francois Richard, Jean-Pierre Desclés, Valérie Flageul. Sémantique cognitive de l'action: 2. étude expérimentale de la catégorisation des verbes d'action. Langages, 1998, 32 (132), pp.48-69. hal-01503800

HAL Id: hal-01503800

https://hal-univ-paris8.archives-ouvertes.fr/hal-01503800

Submitted on 7 Apr 2017

HAL is a multi-disciplinary open access archive for the deposit and dissemination of scientific research documents, whether they are published or not. The documents may come from teaching and research institutions in France or abroad, or from public or private research centers.
L'archive ouverte pluridisciplinaire HAL, est destinée au dépôt et à la diffusion de documents scientifiques de niveau recherche, publiés ou non, émanant des établissements d'enseignement et de recherche français ou étrangers, des laboratoires publics ou privés. 


\title{
Persée
}

http://www.persee.fr

\section{Sémantique cognitive de l'action : 2. étude expérimentale de la catégorisation des verbes d'action}

\author{
C. Kekenbosch;J.-M. Meunier;J.-F. Richard;J.-R Desclés;V. Flageul
}

Langages, Année 1998, Volume 32, Numéro 132

p. $48-68$

\section{Voir l'article en ligne}

The hypothesis investigated is that the semantic similarities of verbs of action are organized according to a limited number of semantic fields and the experiment is designed to identify these fields. Seven subjects have been asked to classify 172 verbs of action, chosen as a function of their frequency of occurrence, so that the verbs belonging to the same class have a definite similarity in meaning, with the possibility for a verb to be classified into several classes. A similarity index between each pair of verbs has been defined as the number of times both verbs of the pair have been ascribed to the same class, and using multidimensional methods six semantic fields have resulted from the analysis : movement and change in place, possession and transfer of possession, modification of a property of an entity or construction of a new entity by fitting parts together, causing damage to an entity by attack or destruction, basic activities neces- sary for life, processes describing the course of action. These semantic groupings have proved to be quite stable in a replication of the experiment using the same subjects.

\section{Avertissement}

L'éditeur du site «PERSEE » - le Ministère de la jeunesse, de l'éducation nationale et de la recherche, Direction de l'enseignement supérieur, Sous-direction des bibliothèques et de la documentation - détient la propriété intellectuelle et les droits d'exploitation. A ce titre il est titulaire des droits d'auteur et du droit sui generis du producteur de bases de données sur ce site conformément à la loi n`98-536 du 1er juillet 1998 relative aux bases de données.

Les oeuvres reproduites sur le site «PERSEE » sont protégées par les dispositions générales du Code de la propriété intellectuelle.

Droits et devoirs des utilisateurs

Pour un usage strictement privé, la simple reproduction du contenu de ce site est libre.

Pour un usage scientifique ou pédagogique, à des fins de recherches, d'enseignement ou de communication excluant toute exploitation commerciale, la reproduction et la communication au public du contenu de ce site sont autorisées, sous réserve que celles-ci servent d'illustration, ne soient pas substantielles et ne soient pas expressément limitées (plans ou photographies). La mention Le Ministère de la jeunesse, de l'éducation nationale et de la recherche, Direction de l'enseignement supérieur, Sous-direction des bibliothèques et de la documentation sur chaque reproduction tirée du site est obligatoire ainsi que le nom de la revue et- lorsqu'ils sont indiqués - le nom de l'auteur et la référence du document reproduit.

Toute autre reproduction ou communication au public, intégrale ou substantielle du contenu de ce site, par quelque procédé que ce soit, de l'éditeur original de l'oeuvre, de l'auteur et de ses ayants droit.

La reproduction et l'exploitation des photographies et des plans, y compris à des fins commerciales, doivent être autorisés par l'éditeur du site, Le Ministère de la jeunesse, de l'éducation nationale et de la recherche, Direction de l'enseignement supérieur, Sous-direction des bibliothèques et de la documentation (voir http://www.sup.adc.education.fr/bib/). La source et les crédits devront toujours être mentionnés. 
Christiane KeKenbosch*, Jean-Marc MeUniER**, Jean-François RiChaRD**, Jean-Pierre DESCLÉS***, Valérie FlaGEUL***

* Groupe de Recherche sur la Parole, Université Paris 8

** Cognition et Activités finalisées. CNRS ESA 7021, Université Paris 8

*** Centre d'Analyse et de Mathématiques Sociales, CNRS / EHESS, Univer-

sité de Paris-Sorbonne

\section{SÉMANTIQUE COGNITIVE DE L'ACTION : 2. ÉTUDE EXPÉRIMENTALE DE LA CATÉGORISATION DES VERBES D'ACTION}

\section{Rationnel de l'expérience}

Nous considérons que, pour identifier un champ sémantique, les significations des verbes appartenant au champ doivent être perçues par les sujets comme proches et comme plus voisines que les significations de verbes appartenant à un autre champ. Notre démarche consiste à repérer d'abord les champs sémantiques à partir de la perception des similitudes de signification qu'ont les sujets des différents verbes, et ensuite à chercher à rendre compte des significations portées par les verbes qui se révèlent proches.

La raison pour laquelle nous cherchons d'abord à identifier les champs, et ensuite à caractériser leur structure sémantique, est la suivante : nous ne sommes pas en mesure actuellement de faire des hypothèses précises sur le contenu sémantique des champs de base, hormis ceux du déplacement et de la possession. Les autres champs sont beaucoup moins conceptualisés et beaucoup plus difficiles à appréhender. Nous avons donc de meilleures chances de les identifier, s'ils existent, en utilisant des méthodes comportementales de nature plus indirecte, comme celles qui utilisent l'activité de catégorisation, et qui sont mieux à même d'appréhender des connaissances non conceptualisées.

Les données actuelles ne permettent pas d'énoncer des hypothèses précises quant au contenu sémantique de ces champs (hormis ceux du déplacement et de la possession). C'est pourquoi nous cherchons d'abord à identifier les champs en utilisant l'activité de catégorisation, méthode qui nous semble-t-il est la mieux adaptée pour appréhender des connaissances implicites. Nous avons utilisé une procédure et une méthode d'analyse analogues à celles de Miller (1969) dans son étude des similitudes entre noms désignant des objets : classement de mots, construction d'une matrice de similitude à partir de ces classements, et étude de la matrice par une méthode d'analyse des proximités qui aboutit à une représentation des similitudes sous forme d'une structure en arbre.

S'il est vrai que c'est l'environnement actanciel qui fixe le sens d'un verbe, il est pratiquement impossible d'étudier un nombre suffisant de verbes au niveau de la prédication, car le nombre de prédications qu'il faudrait considérer serait énorme. Par ailleurs, ce qui nous intéresse ici est l'organisation des significations portées par un ensemble suffisant de verbes pour permettre d'identifier les grands domaines de 
signification. En dépit du fait qu'un verbe donné risque de ne pas être considéré par tous les sujets selon la même acception, nous pouvons raisonnablement supposer que lors d'une tâche de classement les significations activées sont pour l'essentiel celles que les verbes ont en commun.

Ainsi, si un sujet classe le verbe monter avec le verbe descendre, et ces deux verbes avec les verbes aller et venir, la signification de monter qui est activée est celle de déplacement plutôt que celle de construction. Que la signification d'assemblage que possède le verbe monter (dans le sens " monter une voiture, un film, une maison ") ne soit pas évoquée dans ce contexte n'a guère d'importance, car il y a bien d'autres verbes qui portent cette signification d'une façon plus pure, comme assembler, réunir, attacher.

Une tâche de classement, qui privilégie les significations communes, permet de filtrer les significations qui sont caractéristiques du champ. Par ailleurs, ces significations suffisent à la définition des champs, car les significations non évoquées de certains verbes sont des significations évoquées par d'autres verbes : il suffit de prendre un nombre de verbes suffisant pour être assuré de cerner l'ensemble de ces significations. Le fait de pouvoir travailler au niveau des items verbaux constitue donc une simplification considérable de l'investigation et ramène cette dernière à un niveau de complexité qui devient traitable expérimentalement.

Pourquoi choisir une tâche de catégorisation plutôt qu'une tâche de jugement d'attribution de traits ou qu'une tâche d'estimation de similarité entre des verbes pris deux à deux? Une tâche de jugement d'attribution de traits suppose qu'on ait préalablement défini ces traits. Or on ne sait pas quels sont, du point de vue psychologique, les traits qui sont pertinents pour caractériser les significations des verbes, à moins que l'on s'en tienne à des traits très généraux comme statique, dynamique, agentif, intentionnel etc. Demander aux sujets un jugement sur des traits, c'est faire implicitement l'hypothèse que la signification d'un verbe résulte d'une composition additive de traits, ce que précisément nous contestons.

L'autre option serait de faire estimer la similarité de couples de verbes. Cette approche est peu appropriée pour plusieurs raisons. En premier lieu, le nombre de couples que l'on peut construire à partir d'un ensemble de verbes croît très vite : ce nombre est n(n-1)/2, si n est le nombre de verbes. Cela exclut de considérer plus d'une quarantaine de verbes, ce qui est bien insuffisant pour cerner les significations de base des verbes d'action. En second lieu, en donnant à juger les verbes par couples, on introduirait beaucoup de bruit relativement à la définition des champs sémantiques. Lorsque l'on fait comparer deux verbes par un sujet, on introduit beaucoup plus de variabilité pour cette comparaison que lorsque le sujet est amené à comparer ces deux verbes à un même troisième, comme il le fait dans un classement. De plus, cette variabilité affecte directement l'indice de similitude : ainsi, monter aura un degré de similitude intermédiaire par rapport à descendre et à construire : cette position intermédiaire exprime mal le fait que monter a, selon le contexte, soit une signification proche de celle de descendre, soit une signification proche de celle de assembler. Cette double acception est appréhendée d'une façon très directe dans une tâche de classement : monter pourra être classé aussi bien avec descendre qu'avec assembler si le sujet a la possibilité d'intégrer un même verbe à plusieurs classes. 
Il nous paraît donc préférable d'inférer les relations de proximité sémantique des données d'une tâche de classement, plutôt que d'essayer de les obtenir directement à partir d'une tâche de jugement. A partir des relations de similitude ainsi obtenues, nous rechercherons, à l'aide de méthodes d'analyse typologique, quelle est l'organisation de ces significations. L'hypothèse de l'existence de champs sémantiques sera confirmée si, d'une part, il apparaît une structure dans laquelle il y a des coupures nettes entre de grands groupes de verbes, se marquant par une forte similitude par rapport aux éléments du même groupe et une similitude faible par rapport aux éléments des autres groupes, et si, d'autre part, les relations de proximité à l'intérieur des groupes et les coupures entre les groupes sont interprétables comme la manifestation de schémas cognitifs communs et de leurs spécifications.

\section{Expérience 1}

\section{Dispositif expérimental}

\section{Matériel}

Le matériel était composé de 172 verbes français exprimant une action choisis parmi les plus fréquents (d'après Juilland, Brodin et Davidovitch, 1970). Nous avons retenu seulement les verbes exprimant une action dont le résultat est un effet physique et observable et écarté ceux qui présentaient une polysémie manifeste. La liste complète des verbes est donnée en annexe 1.

\section{Sujets}

Sept sujets, étudiants ou universitaires de Paris 8 , ont participé à l'expérience.

\section{Procédure expérimentale}

Le sujet avait à ranger les verbes en catégories ; il avait en face de lui, sur la gauche de l'écran d'un ordinateur Macintosh, la liste des verbes, rangés par ordre alphabétique, et à droite une vingtaine de cases destinées à ranger les verbes. On montrait au sujet comment copier un verbe dans une case, en cliquant successivement avec la souris sur le verbe et sur la case correspondant à la classe où il souhaitait placer le verbe.

La consigne était la suivante :

Vous avez sur la gauche une liste de verbes. Vous devez mettre ensemble les verbes qui, selon vous, s'accordent par le sens, en utilisant pour cela les cases dessinées sur la droite. Par exemple, si vous voulez mettre ce verbe dans cette classe, vous cliquez sur le verbe et vous cliquez sur la classe (deux ou trois verbes sont copiés dans differrentes cases à titre d'exemples). Votre tâche est de continuer la classification. Si un verbe d'une case ne vous paraît pas aller avec les autres, vous pouvez le mettre ailleurs. Vous pouvez faire autant de classes que vous voulez, en utilisant les cases vides. Vous pouvez mettre le même verbe dans plusieurs cases. S'il y a des verbes que vous ne savez pas où classer, mettez-les dans une case "divers". Avant de commencer la classification, lisez la totalité de la liste. Vous pouvez modifier votre classement à tout moment 
si vous n'êtes pas satisfait. Quand vous aurez fini, essayez de trouver pour chaque case un terme général qui la désigne le mieux.

Aucune limite de temps n'était imposée. L'expérience durait approximativement deux heures.

\section{Résultats}

À partir des classes produites par les sujets, nous avons défini un indice de similarité pour toutes les paires de verbes ; le score de similarité d'une paire est le nombre de fois où les deux verbes ont été rangés dans une même classe par un sujet : la valeur maximum de cet indice est 7 , soit le nombre de sujets.

Une condition pour l'existence d'une catégorie sémantique, c'est le fait que les verbes qui sont dans la catégorie sont fortement associés à chacun des autres et sont faiblement associés aux verbes des autres catégories. Dans un premier temps, nous avons considéré que deux verbes sont fortement associés s'ils ont été mis dans la même classe par une majorité de sujets, c'est-à-dire au moins quatre sujets sur sept. Si nous retenons dans la matrice de similarité les scores de similarité de quatre ou plus et si nous mettons ensemble les éléments qui sont connectés les uns aux autres, huit catégories distinctes apparaissent. Un certain nombre de verbes, comme " prendre ", appartiennent à plusieurs groupes. Ces catégories ne sont donc pas toutes indépendantes entre elles, certains verbes appartenant à plusieurs classes. Un verbe appartient à une catégorie s'il est connecté à au moins un verbe de la catégorie. Bien sûr, le degré de liaison entre les verbes d'une catégorie est variable : quelques verbes, qui sont connectés à beaucoup d'autres, seront considérés comme représentatifs de la catégorie. Nous avons défini un indice de cohésion de la catégorie en calculant la moyenne des scores de similarité entre chaque paire de verbes de la catégorie. Dans le tableau 1 sont présentés, pour chaque catégorie, les verbes les plus représentatifs et l'indice de degré de cohésion à l'intérieur de la catégorie (les catégories complètes sont données en annexe 2). On peut ainsi dégager huit groupes de signification.

\begin{tabular}{|l|c|c|l|}
\hline \multicolumn{1}{|c|}{ catégories } & $\begin{array}{c}\text { nombre } \\
\text { de verbes }\end{array}$ & $\begin{array}{c}\text { degré de } \\
\text { cohésion }\end{array}$ & \multicolumn{1}{|c|}{ verbes les plus représentatifs } \\
\hline $1:$ mouvement & 39 & 3,15 & traverser, venir, parcourir, avancer, aller \\
\hline 2: déplacement & 28 & 2,01 & emmener, emporter, porter, déplacer \\
\hline 3: possession & 18 & 3,11 & donner, perdre, prêter, rendre, offrir \\
\hline $\begin{array}{l}4: \text { changement } \\
\text { de propriété }\end{array}$ & 25 & 1,57 & $\begin{array}{l}\text { déformer, agrandir, changer, écarter, } \\
\text { augmenter }\end{array}$ \\
\hline $5:$ assemblage & 28 & 1,79 & $\begin{array}{l}\text { assembler, placer, mettre, fixer, attacher, } \\
\text { rapprocher }\end{array}$ \\
\hline $\begin{array}{l}\text { 6: dissociation } \\
\text { du tout en parties }\end{array}$ & 15 & 1,82 & détruire, briser, rompre, casser, couper \\
\hline $7:$ agression & 20 & 2,00 & battre, attaquer, frapper, combattre, éviter \\
\hline 8: processus & 34 & 1,93 & finir, faire, se mettre à, commencer, terminer \\
\hline
\end{tabular}

Tableau 1 : les verbes les plus représentatifs par catégorie 
Dans un second temps, afin d'affiner notre analyse, nous avons procédé à une analyse hiérarchique en utilisant la méthode des scores (Barthélémy et Guénoche, 1988). Le principe de la méthode est le suivant : étant donné quatre points $i, j, k$ et 1 , on peut faire une bipartition de ces points en deux paires $(i, j)$ et $(k, 1)$ telle que la somme des distances entre les deux points d'une même paire $[D(i, j)+D(k, l)]$ soit inférieure (ou égale) à la somme des distances entre chaque point d'une paire et chaque point de l'autre $[D(i, k)+D(j, l))]$ ou $[D(i, l)+D(j, k)]$. On définit le score d'une paire $(i, j)$ comme étant, sur l'ensemble des quadruplets possibles, le nombre de quadruplets pour lesquels la paire $(i, j)$ est l'une des paires de la partition. Une paire qui a un score élevé est donc une paire dont les deux points sont proches. On regroupe les points de la paire dont le score est maximum et on crée un nouveau point dont la similarité aux autres points est la moyenne des similarités des deux points de la paire aux autres points. On continue en regroupant les deux points dont le score est maximum. Nous avons utilisé le programme de Y. Guénoche (que nous remercions d'avoir mis gracieusement son programme à notre disposition). On obtient un arbre valué dont les feuilles terminales sont les verbes, et les nœuds, numérotés dans l'ordre de groupement, sont les classes de différents niveaux. Dans ce type d'arbre, la distance verticale entre les nouds est proportionnelle à la distance entre les classes (les distances horizontales n'ont pas de signification).

Dans la mesure où la structure de nos données est plus proche d'un treillis que d'un arbre, nous avons également procédé à une seconde analyse en éliminant les verbes qui appartiennent à plusieurs des classes identifiées dans la première analyse hiérarchique. Il serait certes préférable d'utiliser une méthode qui donne une représentation sous forme de treillis, mais nous n'avons pas considéré de telles méthodes, car d'une part elles sont de nature combinatoire et d'autre part elles ne sont pas opérationnelles pour de grands ensembles de données.

Nous retiendrons comme critère d'appartenance à une classe une fréquence moyenne d'association aux verbes de chacune des classes identifiées dans la première analyse hiérarchique, le degré de cohésion le plus faible observé dans la première analyse, soit 1,5 . Les verbes qui ont une fréquence d'association moyenne supérieure pour plus d'une classe seront écartés de l'analyse.

La figure $1 a$ (p. 53) présente les premiers niveaux de la classification hiérarchique pour l'ensemble des 172 verbes. Chaque classe est désignée par le nom d'une notion associée aux significations des verbes de la classe. Nous donnerons plus loin une analyse plus précise des contenus sémantiques de ces classes.

On voit que se dégagent six grandes classes de signification, que l'on peut désigner ainsi :

- possession

- activités de base

- agression et dissociation du tout en parties

- assemblage et modification des propriétés d'un objet

- verbes exprimant des processus

- mouvement et déplacement

Dans ces six catégories, on retrouve les huit classes de la première analyse, mais les classes mouvement et déplacement d'une part, et les classes assemblage et modification de propriétés d'autre part, se trouvent intégrées dans une seule classe. 


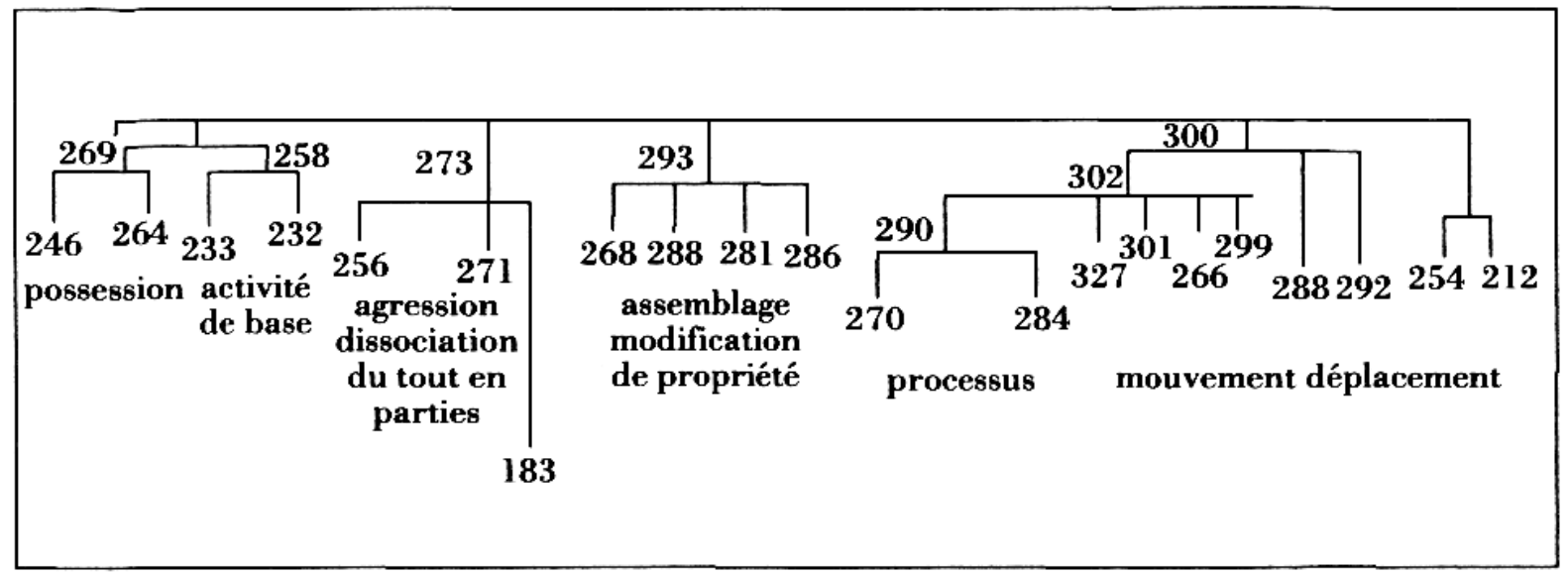

Figure 1a : Analyse hiérarchique de l'ensemble des 172 verbes

Pour la seconde analyse hiérarchique, nous avons calculé, pour chaque verbe, à partir du tableau original des similarités, le nombre moyen de fois où il se trouve associé aux verbes de chacune des six classes. On a ainsi un indice d'association de chaque verbe à chacune des six classes. Nous avons éliminé tous les verbes ayant un indice supérieur à 1,5 (voir plus haut) pour plus d'une classe. Il reste 112 verbes qui sont donnés en annexe. On notera que le nombre des verbes qui se trouvent liés à plusieurs classes est important. Nous avons alors appliqué à ces 112 verbes l'analyse hiérarchique basée sur la méthode des scores. Les résultats sont présentés dans la figure lb.

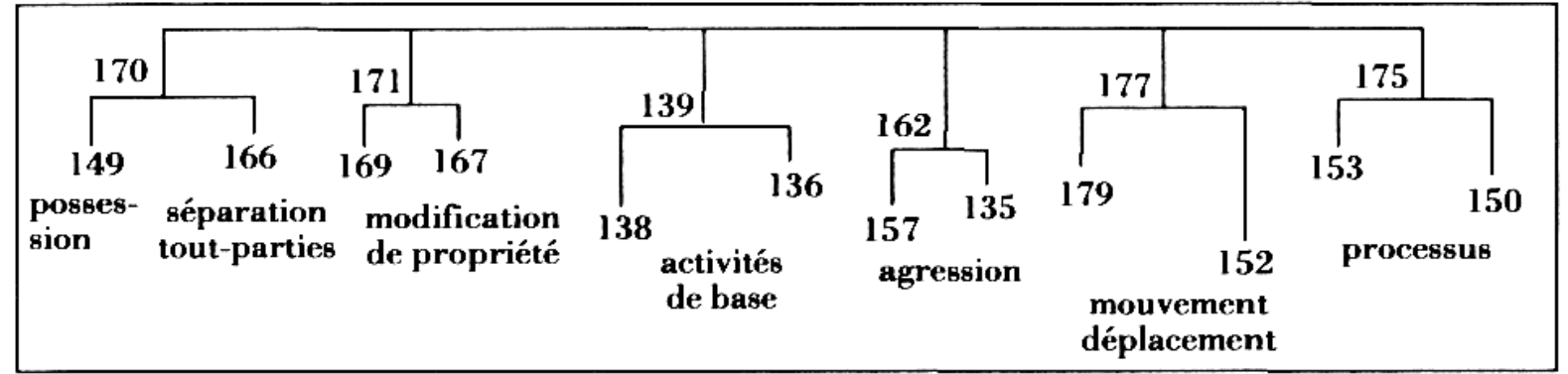

Figure 1 b : Analyse hiérarchique après élimination des verbes appartenant à plusieurs catégories

Dans cette analyse on trouve sept classes nettement différenciées. Parmi ces sept classes, quatre restent identiques aux résultats de l'analyse précédente. Ce sont :

- possession

- activités de base

- mouvement-déplacement

- verbes exprimant des processus

Une classe est dissociée en deux : c'est la classe dénommée " agression, dissociation du tout en parties». Les deux classes apparaissent nettement disjointes dans la seconde analyse, alors qu'elles étaient regroupées dans la première analyse, tout en étant identifiables, sous le noud 273 . Une classe se trouve caractérisée un peu différemment : c'est la classe dénommée « assemblage et modification de propriétés d'un objet ». La nouvelle classe ne comporte que des verbes exprimant une modification de propriétés d'un objet. Cela s'explique par le fait que tous les verbes exprimant un assemblage ont été supprimés parce qu'ils se trouvent appartenir à plusieurs classes. En effet ces verbes appartiennent également en général à la classe " mouvement- 
déplacement ". Les différences entre les résultats des deux analyses restent mineures, bien que le tiers des verbes ait été retiré dans la seconde analyse.

Nous allons maintenant analyser de plus près les classes issues de la première analyse.

- La classe 269 (possession) est subdivisée en deux sous-classes,

- 246 : qui inclut les verbes exprimant le transfert de possession (rendre, donner, prêter, offrir, acheter, etc.)

- 264 : qui contient les verbes exprimant la conservation de la possession (garder, tenir, maintenir, conserver, etc.)

- La classe 258 (activités de base) est composée de deux groupes :

- le groupe 232 qui comporte des verbes tels que respirer, manger, boire, nourrir, endormir ;

- le groupe 233 qui comporte des verbes comme pleurer, crier, murmurer, trembler, jouer, chanter.

Le premier groupe exprime les activités de base du vivant, le second semble désigner des activités plus spécifiquement humaines en ce qu'elles apparaissent liées à des sentiments.

- La classe 273 regroupe d'une part des verbes exprimant l'agression comme attaquer, tuer, combattre et d'autre part des verbes signifiant la dissociation d'un objet en parties comme rompre, couper, briser. Ce sont deux groupes de signification qui apparaissent dans des classes nettement différenciées dans la seconde analyse hiérarchique.

- La classe 293 comporte deux sous-classes assez facilement interprétables : celle des verbes qui expriment un assemblage (268) et celle des verbes qui expriment une modification des propriétés d'un objet (288). Elle comporte également deux groupes moins immédiatement interprétables comportant des verbes comme remuer, agiter, tacher, toucher (281) et des verbes comme préparer et serrer (286).

- La classe 290 contient des verbes exprimant des processus qui décrivent le cours de l'action. On note deux groupes, l'un (284) qui exprime l'interruption d'activité et l'autre (270) qui exprime la réalisation de l'activité ainsi que les phases de son déroulement.

- Sous la classe 300 on trouve plusieurs groupes correspondant au mouvement et au déplacement d'objets. Contrairement à la première analyse, ces deux classes de verbes se trouvent regroupées. D’autres résultats expérimentaux (Meunier, 1992) nous conduisent cependant à conserver la distinction entre ces deux catégories de verbes, dont la proximité dans cette analyse tient probablement à l'opposition de ces deux classes aux autres catégories sémantiques.

\section{Expérience 2}

Pour vérifier la stabilité des résultats de la première expérience, nous avons répété l'expérience. Nous avons retrouvé, trois ans après, cinq des sujets qui ont participé à la première expérience, et qui ont réalisé la tâche de classement dans les mêmes conditions. 


\section{Procédure expérimentale}

Nous avons utilisé la même procédure expérimentale, avec le même matériel et le même programme. Nous avons seulement introduit deux modifications mineures :

a) Au début de l'expérience, aucun début de groupement n'était proposé, vingt cases vides étaient présentées (nombre moyen de cases faites par les sujets dans la première expérience).

b) Nous avons demandé aux sujets de dire à haute voix le sens dans lequel ils comprennent les verbes, en donnant un synonyme ou une courte phrase.

\section{Résultats}

Nous avons utilisé la même procédure d'analyse hiérarchique (méthode des scores). Les résultats sont très proches de ceux de la première expérience. On retrouve les mêmes grandes classes identifiées précédemment.

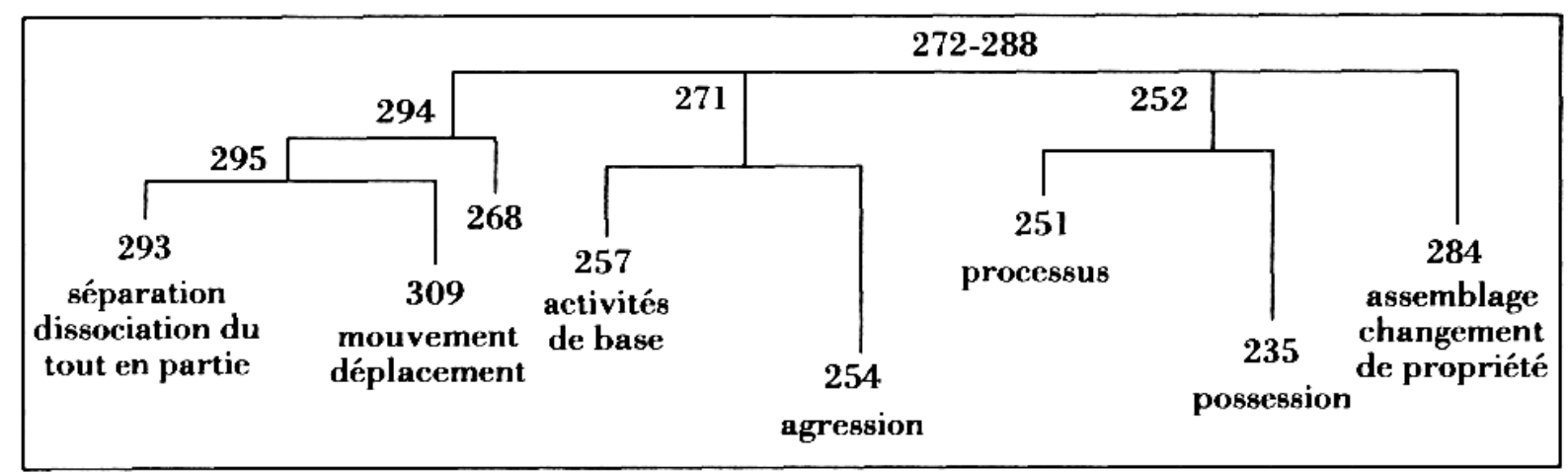

Figure 2 : Analyse hiérarchique des résultats de la deuxième expérience

Si on examine de plus près chacune de ces classes, on peut noter quelques différences d'organisation entre les classes, mais qui ne remettent pas en cause le découpage précédemment établi.

La classe 284 regroupe les verbes exprimant le changement de propriétés d'un objet (tels que peindre, tacher, agrandir) et les verbes d'assemblage (tel que assembler, réunir, attacher), dont on avait déjà vu la proximité dans l'expérience 1. Quelques verbes atypiques de la première expérience tels que remuer, toucher, agiter se trouvent classés ailleurs. En revanche d'autres verbes, tels que allumer, éteindre, dont l'absence parmi les verbes de changement de propriétés pouvait surprendre, apparaissent dans cette deuxième expérience.

Sous la classe 252 , on trouve deux grandes classes :

- sous l'une, la classe 235, nous retrouvons les verbes dénotant la possession. Comme dans la première expérience, on a une branche contenant les verbes relatifs à la conservation de la possession et une autre sous-classe constituée des verbes de transfert de possession. Cinq verbes de la classe des verbes de possession de la première expérience manquent dans cette classe 235 : prendre, tenir, supporter, tenir, perdre. Pour les quatre premiers ce n'est pas étonnant, car ce sont plutôt des verbes exprimant le déplacement. Quant à perdre, il est par son sens un peu à part dans les verbes de transfert de possession.

- sous l'autre branche de la classe 252 , on a la classe 251 , constituée de verbes dénotant l'activité d'un sujet. Dans la première expérience, ces verbes étaient plus 
proches des verbes de mouvement et de déplacement. Le fait de les retrouver, dans cette expérience, proches des verbes de possession est un argument en faveur de l'autonomie de ce groupe de signification par rapport aux verbes exprimant un mouvement ou un déplacement.

Sous la classe 271 , on distingue deux classes : la classe 254 , constituée des verbes d'agression et la classe 257 où l'on retrouve les verbes dénotant les activités de base du sujet, deux classes qui étaient précédemment séparées.

Deux autres groupes de verbes se trouvent réunis sous la classe 295 : ce sont les classes 309 et 293. La classe 293 contient des verbes dont la signification évoque la destruction d'un objet (briser, rompre, casser) et la dissociation d'un tout en ses parties (séparer, détacher). Sous la classe 309, il apparaît deux sous-classes qui regroupent, l'une, des verbes exprimant le mouvement, l'autre des verbes de déplacement.

En conclusion, nous pouvons constater que même si certains verbes, du fait de leur polysémie, ont changé de classe, les grands groupes de signification de la première expérience sont conservés. Les classes mises en évidence lors de l'expérience sont donc bien des catégories stables en mémoire. Les changements de catégorie sont au total peu importants et s'expliquent aisément : ainsi des verbes comme prendre et saisir passent de la catégorie possession (269) dans la première expérience à la catégorie mouvement/déplacement (300) dans la seconde. D'autres comme allumer ou éteindre passent de la catégorie mouvement/déplacement à la catégorie modification de propriétés d'un objet.

\section{Discussion et analyse linguistique des classes dégagées à partir des données expérimentales}

L'analyse des résultats de la première expérience met en évidence huit classes parmi les verbes d'action. Nous allons analyser ces classifications du point de vue de la Grammaire Applicative et Cognitive afin de dégager pour chacune des classes de verbes le schème prototypique qui la caractérise. Une fois cette analyse effectuée, nous pouvons chercher si la seconde analyse, qui est elle beaucoup plus fine tout en se basant sur le même protocole, confirme les résultats dégagés lors de la première analyse.

L'interprétation d'un item verbal, pris à l'intérieur d'une classe, n'est pas représentative de cet item mais de l'occurrence de cet item dans la classe pour que cette signification soit cohérente avec celle de la classe. Par exemple, un même verbe peut entrer dans plusieurs classes; on retiendra du verbe un point de vue ou un autre. Un verbe admet un réseau de représentations sémantico-cognitives, pour lequel on peut éventuellement déterminer un invariant. Ici nous ne manipulons pas l'invariant des verbes, mais une des significations de leur réseau. Pour une classe donnée, il est possible de retrouver un contexte à chaque verbe, qui illustre la signification adoptée pour la classification. Les catégorisations effectuées par les sujets au cours de l'expérience mettent en évidence des champs sémantico-cognitifs.

\subsection{Interprétation des catégories issues de la première analyse de l'expérience 1}

Nous allons ici examiner une à une les classes dégagées par la première analyse de l'expérience 1, pour en extraire la signification prototypique. Le schème ainsi élaboré donnera alors une justification linguistique aux classes fournies par les sujets. 
La première classe se compose des verbes suivants : traverser, venir, parcourir, avancer, se déplacer, aller. Pour chacun de ces verbes, une entité effectue un mouvement dont elle a le contrôle. Nous proposons donc, pour représenter ce qu'il y a de commun à ces verbes, le schème sémantico-cognitif (S.S.C.) suivant :

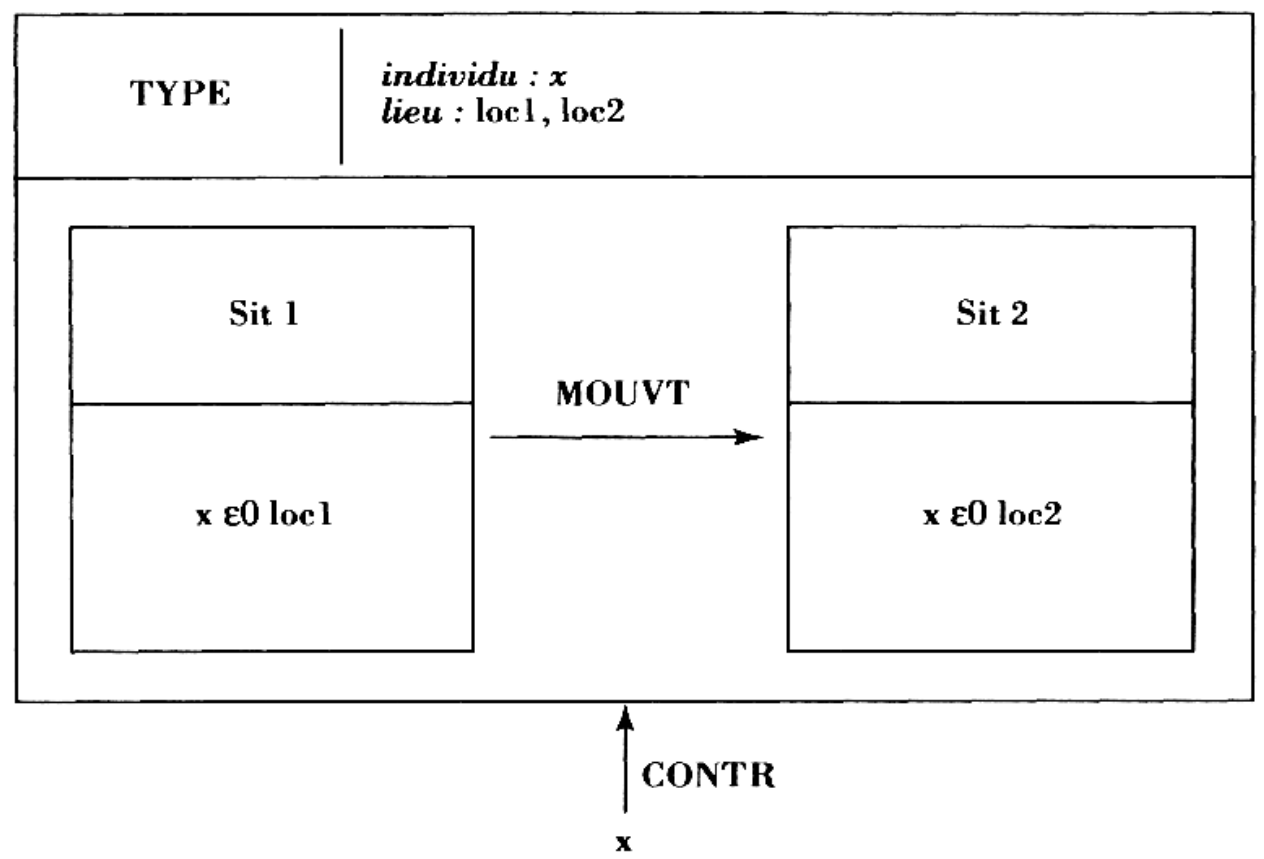

On passe d'une situation initiale Sit 1 où une entité $x$ est repérée par rapport à un lieu locl à une situation Sit 2 où $x$ est repéré par rapport à un lieu loc2. Le mouvement ainsi décrit est sous le contrôle de ce même $x$. Ce schème représente l'invariant de la classe et ne peut donc tenir compte des spécificités de chaque verbe, dont l'étude n'est pas l'objet de cet article.

Les verbes de cette première classe sont caractérisés par un mouvement avec contrôle interne.

La seconde classe est composée des verbes : emmener, emporter, porter, déplacer, soulever. Pour chacun de ces verbes, une entité est déplacée, différente de celle qui contrôle le mouvement. Ceci se représente de la manière schématisée p. 58.

On passe d'une situation Sit 1 où une entité $y$ est repérée par rapport à un lieu loc 1 à une situation Sit 2 où $y$ est repéré par rapport à un lieu loc 2 ; le mouvement ainsi décrit est sous le contrôle de $\boldsymbol{x}$. Cette classe représente un mouvement avec contrôle externe.

Les verbes de la troisième classe sont : donner, perdre, prêter, rendre, offrir. Pour chacun de ces verbes, une entité $x$ est en possession d'une entité $y$ puis perd cette possession.

Dans la situation Sit $1, x$ est en possession de l'entité $y$, puis en Sit $2 x$ n'est plus en possession de $y$. Les verbes de cette classe peuvent être caractérisés par la propriété de transfert. 


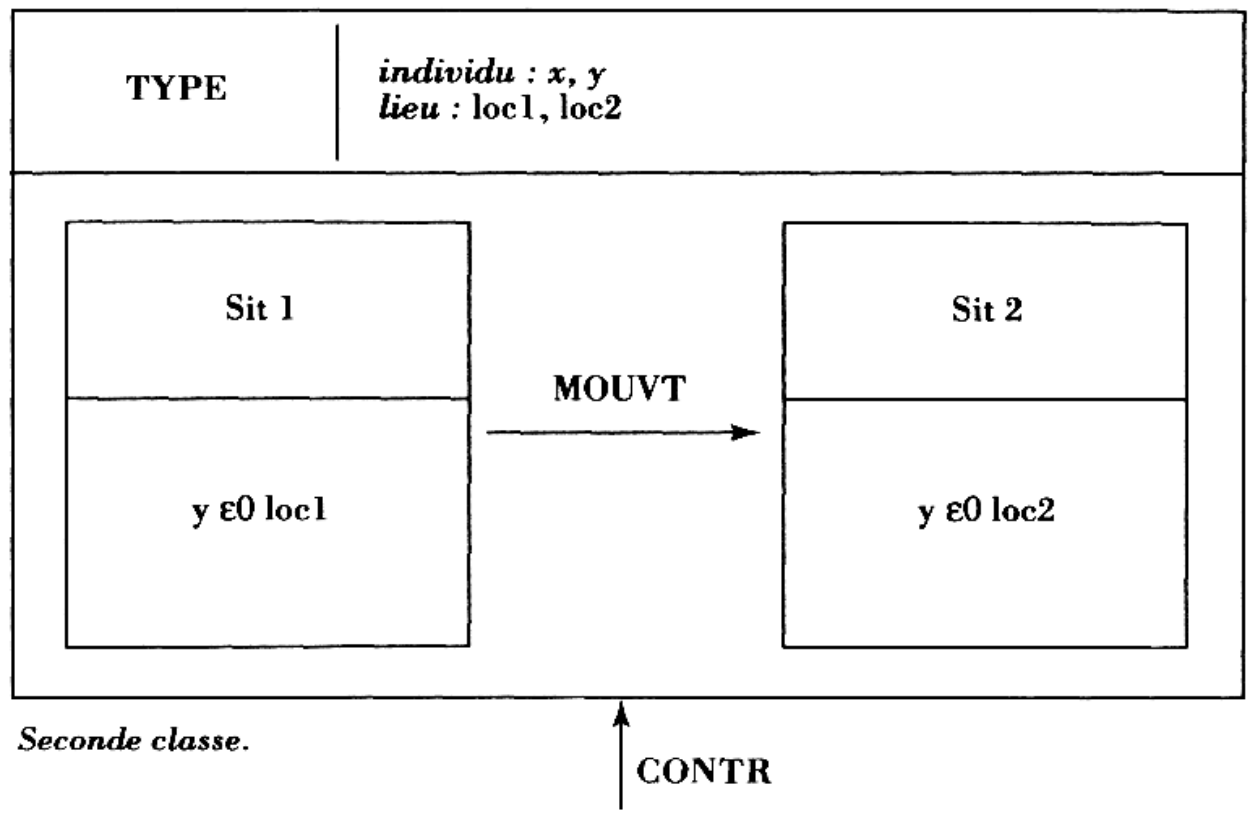

$\mathbf{x}$

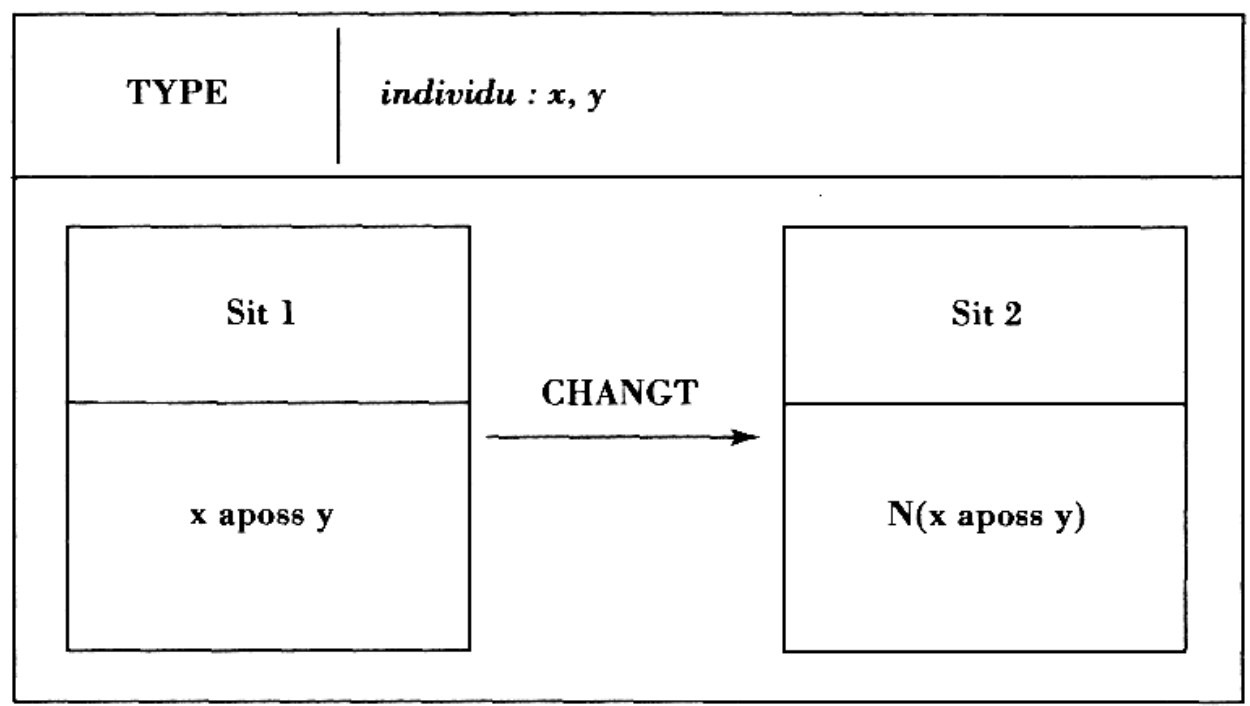

Troisième classe.

La quatrième classe est composée des verbes : déformer, agrandir, changer, écarter, augmenter. Pour chacun de ces verbes, une entité provoque par son action, volontaire ou non, le changement qui affecte une autre entité. Ceci peut se formaliser par le S.S.C. représenté p. 59.

On passe d'une situation Sit 1 où $y$ l'attribut de $\mathrm{z}$ a pour valeur $\alpha$, à une situation Sit 2 où $y$ a pour valeur $\beta$ : ce changement de valeur de l'attribut de $z$ s'effectue avec le faire de $x$. Nous pouvons identifier la classe comme étant représentative d'un changement de valeur de l'attribut d'un objet.

La cinquième classe comprend les verbes : assembler, placer, mettre, fixer, attacher, rapprocher. Avec ces verbes, on observe une mise en rapport des différentes entités. On a alors le schème suivant : 

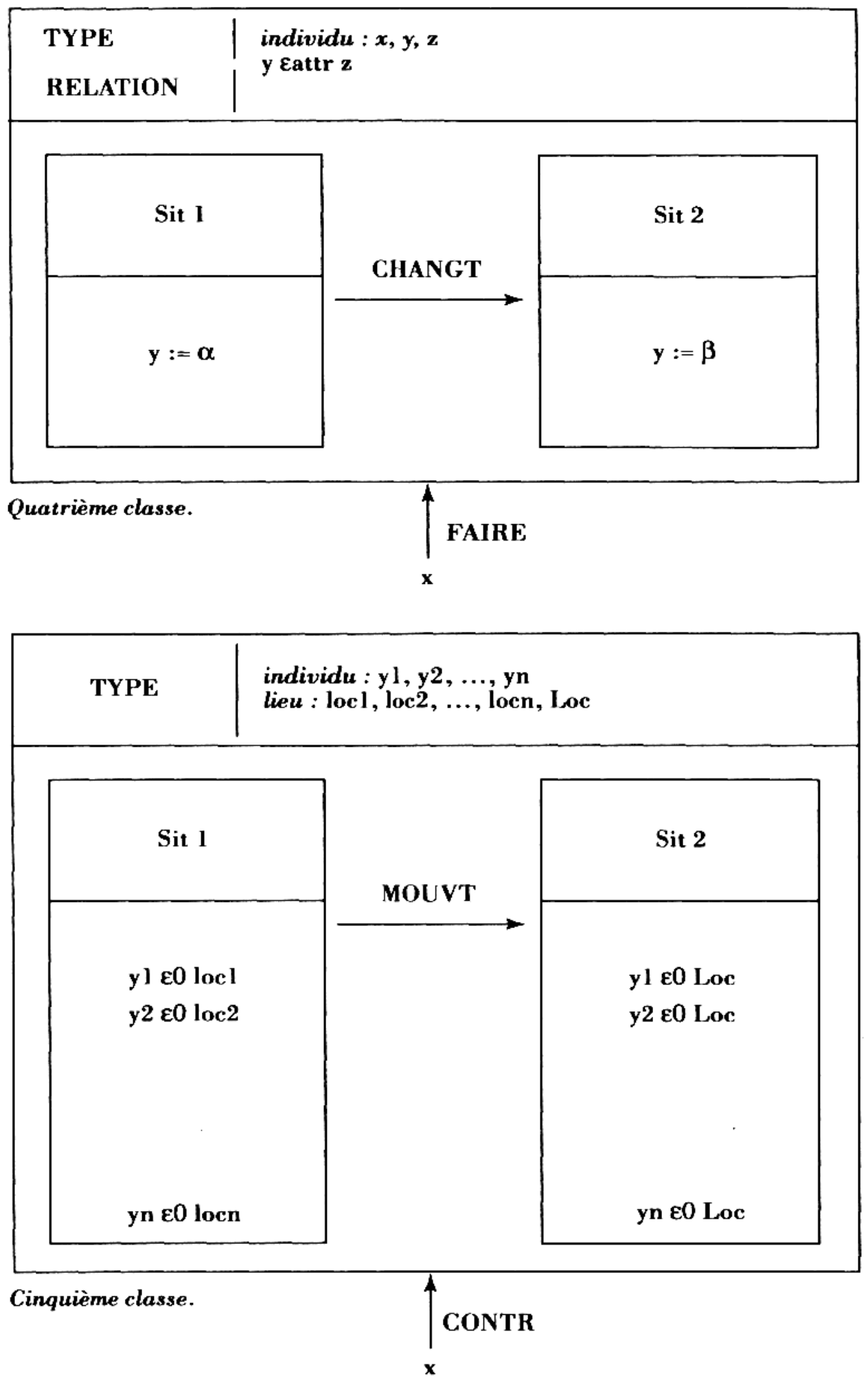

On passe d'une situation Sit 1 où, pour $i$ variant de 1 à n, chaque $y_{i}$ est repéré par rapport à un lieu loc li $_{i}$ non spécifié, à une situation Sit 2 où tous les $y_{i}$, avec $i$ variant de 1 à n, sont repérés par rapport à un unique lieu Loc, ce mouvement étant contrôlé par $x$.

Si les entités ne sont plus repérées globalement par rapport à un même lieu mais l'une par rapport à l'autre, on a alors le schème, qui peut être vu comme une spécification du schème précédent : 


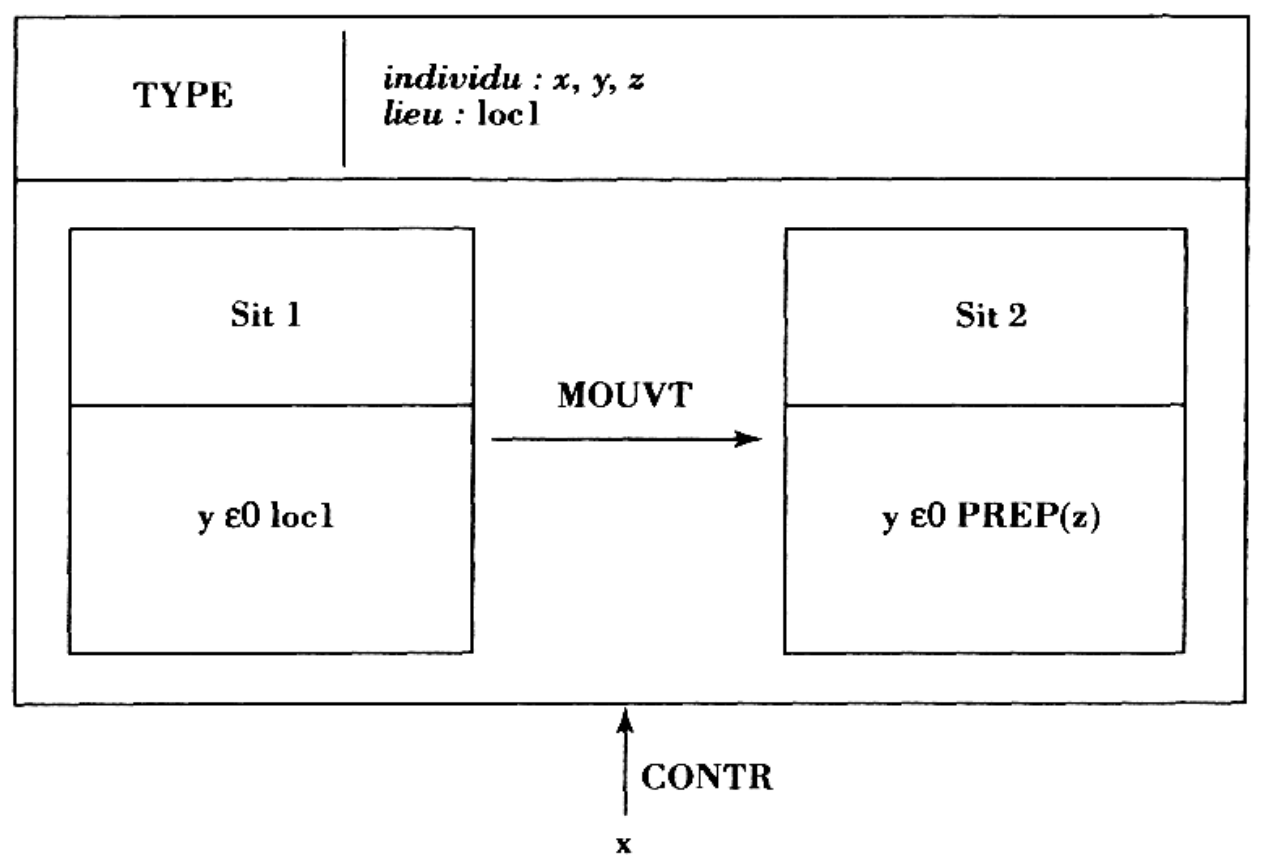

Les verbes de cette classe sont donc caractérisés par le fait de mettre en rapport certaines entités, de structurer la relation qui les unit. Nous nommerons donc la classe : Mouvement organisateur (avec des contraintes).

La sixième classe se compose des verbes : détruire, briser, rompre, casser, couper mais il n'y a pas nécessairement contrôle. Pour représenter cela, nous proposons le schème suivant :

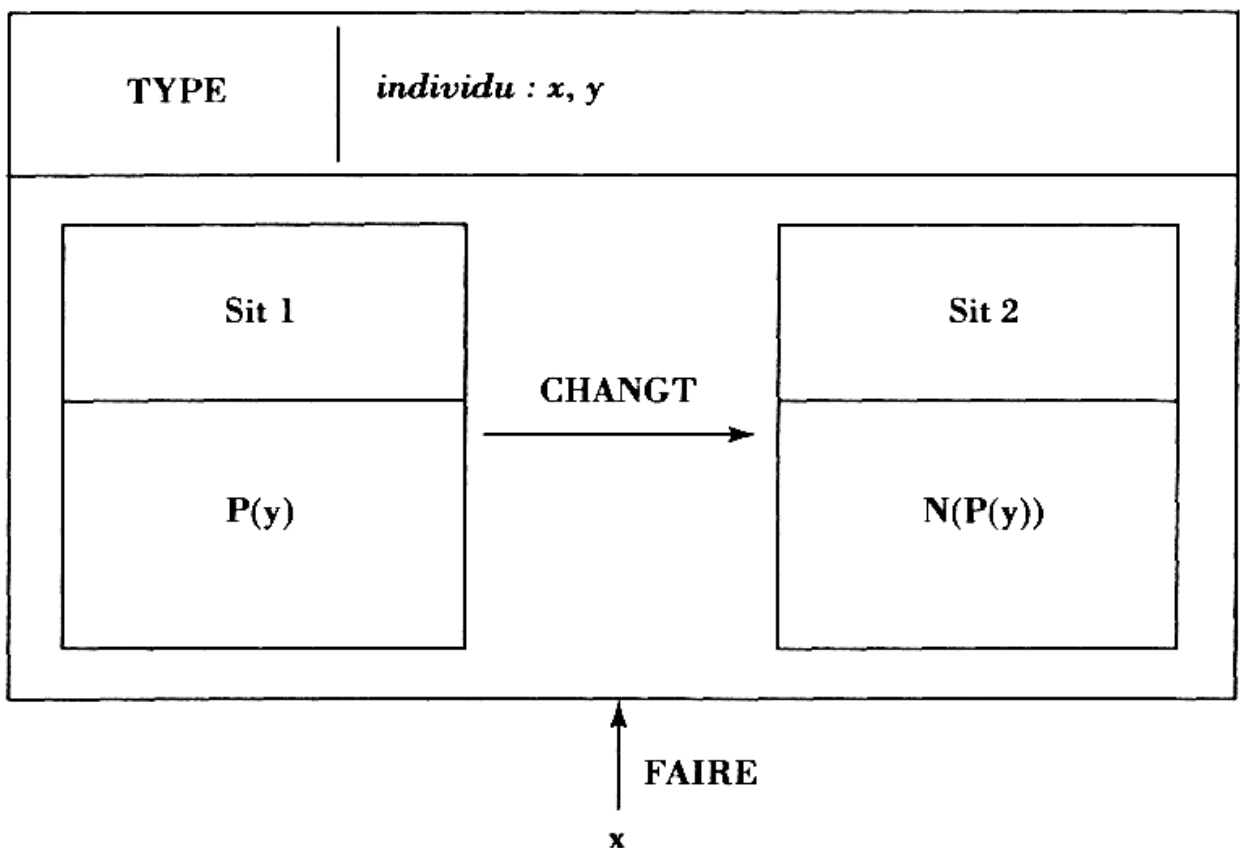

Dans la situation Sit. $1, y$ a la propriété $P$, où $P$ est une propriété constitutive de $y$, alors que dans la situation Sit 2 y n'a plus la propriété $P$; ce changement a lieu avec le faire de $x$. Cette classe est donc caractérisée par la destruction d'une propriété constitutive d'un objet. 
La septième classe concerne les verbes : battre, attaquer, frapper, combattre, éviter. Les quatre premiers impliquent pour une entité d'en agresser une autre, c'est-à-dire de pénétrer dans le lieu propre de l'autre entité sans autorisation ; le verbe éviter par contre implique une représentation de cette agression qui n'a finalement pas lieu.

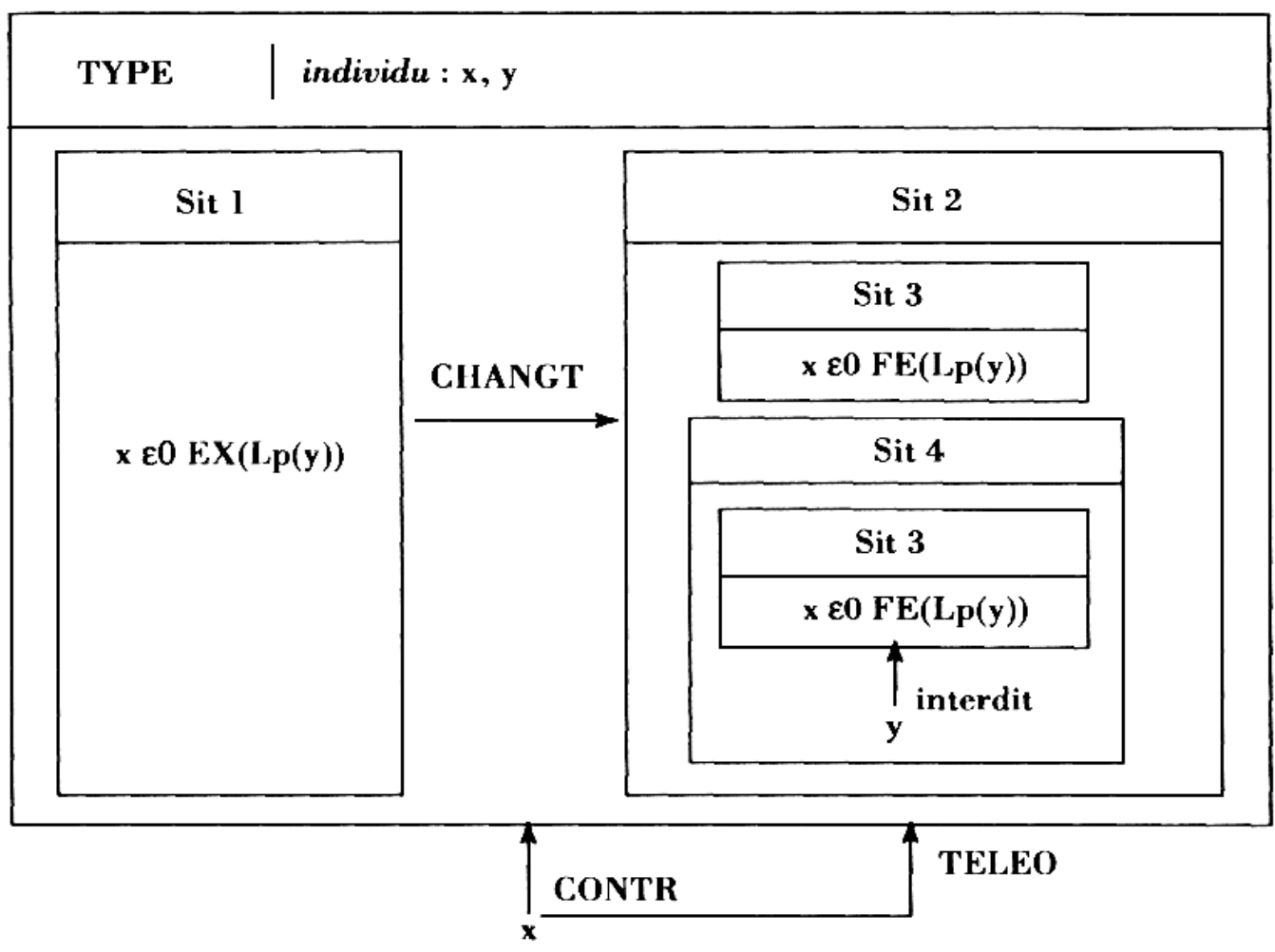

On passe ici d'une situation Sit 1 où une entité $x$ est repérée par rapport à l'extérieur du lieu propre de $y$, l'entité victime de l'agression, à une situation Sit 2 où d'une part $x$ est repéré par rapport à la fermeture du lieu propre de $y$ et d'autre part $y$ interdit cette situation ; ce changement est sous le contrôle de $x$. De plus $x$ vise la situation Sit 2 ; il a donc pour but de pénétrer dans la fermeture du lieu propre de $y$ tout en sachant que $y$ l'interdit.

Le schème correspondant au verbe éviter est représenté p. 62.

On passe ici d'une situation Sit 1 où une entité $x$ est repérée par rapport à l'extérieur du lieu propre de $y$, tout en se représentant qu'elle va heurter l'entité $y$, c'est-à-dire qu'elle va passer de l'extérieur du lieu propre de $y$ à sa fermeture, à une situation Sit 2 où $x$ est repéré par rapport à l'extérieur du lieu propre de $y$; ce changement est sous le contrôle de $x$. $x$ vise la situation Sit 2 , tout en se représentant la situation Sit 7 comme devant se produire s'il n'y avait pas eu changement.

Nous pouvons nommer la classe agression puisque dans ces deux schèmes il est question d'entrer ou non dans le lieu propre de l'autre.

La dernière classe comprend les verbes : finir, faire, se mettre à, commencer, terminer regroupés pour leurs propriétés aspectuelles, non pour leurs propriétés lexicales. 


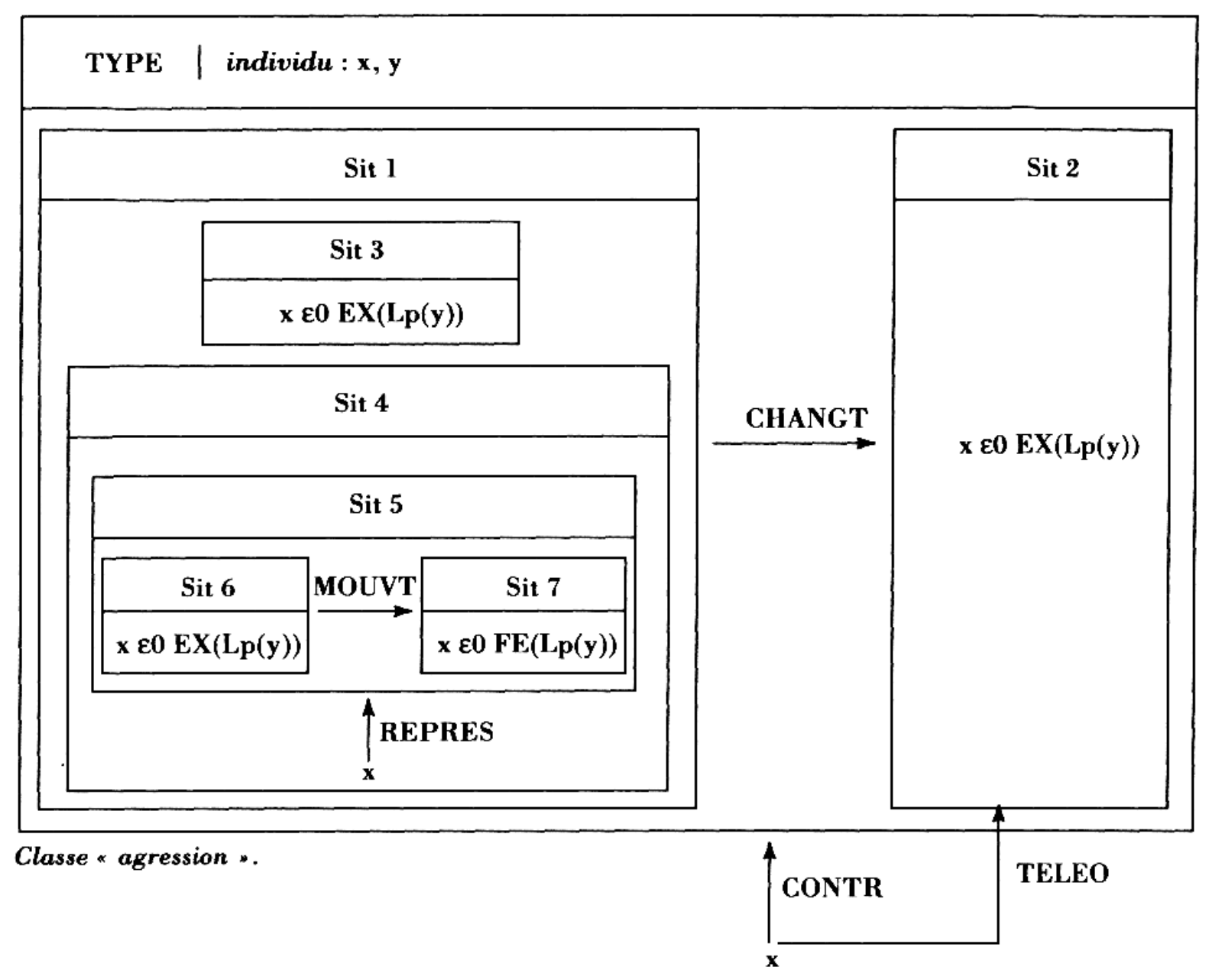

Nous allons ici considérer des entités de type « activité » qui peuvent engendrer des lieux d'activité (L.AC), auxquels nous pouvons appliquer les opérateurs topologiques (Abraham 1995). On peut représenter les verbes listés ci-dessus sur le diagramme suivant :

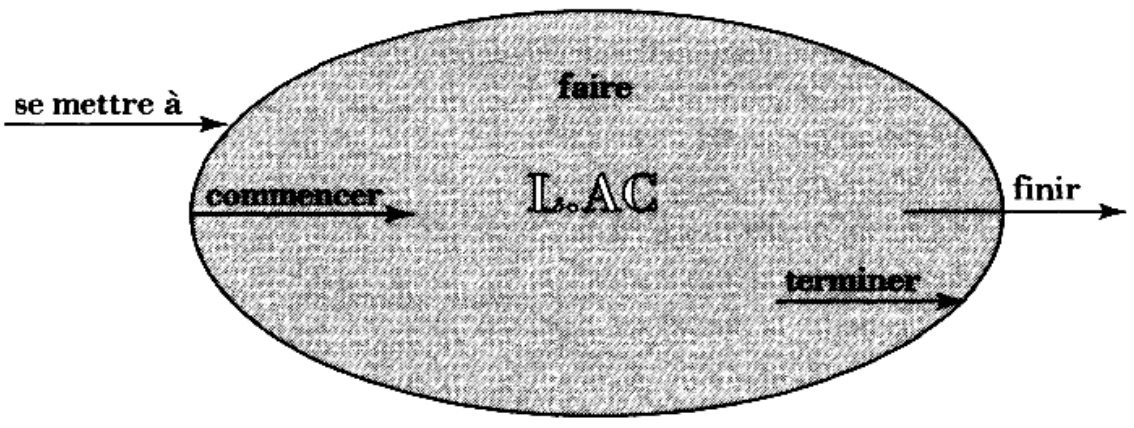

Ainsi le verbe se mettre à correspond au passage de l'extérieur d'un lieu d'activité à sa frontière ; terminer correspond au passage de l'intérieur d'un lieu d'activité à sa frontière, finir au passage de l'intérieur à l'extérieur d'un lieu d'activité, etc. Nous pouvons donner à titre d'exemple le SSC correspondant au verbe commencer (Abraham, 1995) - cf. p. 63.

On passe donc d'une situation Sit 1 où $x$ (variable de situation) est repéré par rapport à la frontière du lieu d'activité $L$.ACn, à une situation Sit 2 où $x$ est repéré par rapport à la fermeture de l'intérieur de L.AC. Pour décrire les autres verbes à 


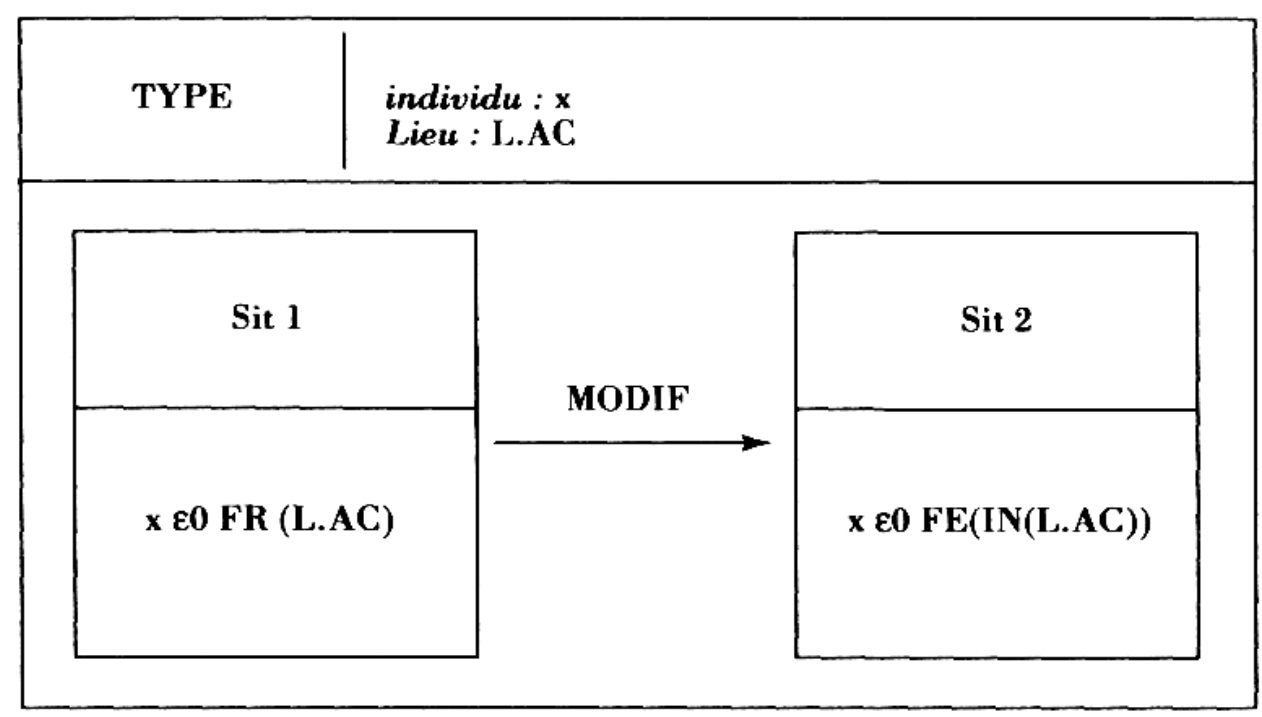

propriété aspectuelle nous pourrons utiliser les opérateurs de l'algèbre de Kuratowski. On nommera la classe : Processus décrivant une activité.

\subsection{Interprétation linguistique des classes issues de l'analyse hiérarchique}

La classe 269 comprend tous les verbes présents dans la classe que nous avions nommée " transfert". Elle comprend par ailleurs un certain nombre de verbes qui étendent la classe et qui de ce fait n'a plus comme unité la notion de transfert mais la notion plus générale de possession.

La classe 258 n'apparaît que dans la seconde analyse ; les fréquences d'association ne sont pas très fortes, ce qui explique qu'on ne retrouve pas cette classe dans la première analyse (qui était fondée uniquement sur le critère de fréquence d'association). Les verbes de la sous-classe 233 sont l'expression d'un état interne : les verbes de la sous-classe 232 font intervenir un autre actant qui est relatif à un état interne.

La classe 273 reprend entre autres les verbes des classes de l'agression et de la destruction d'une propriété constitutive d'un objet. On y distingue alors trois sousclasses : les deux mentionnées ci-dessus plus une sous-classe qui comprend des verbes de « désassemblage ». Ce qui caractérise les verbes de cette classe est qu'on agit sur l'intégrité d'un objet ou d'une personne. Le schème 6 peut alors être un schème prototypique adéquat pour l'ensemble de la classe, le schème de la classe 7 étant alors une spécification du schème de la classe 6 dans laquelle on précise ce que c'est que l'intégrité d'une personne. En ce qui concerne la propriété de désassemblage, c'est la relation de partie-tout qui constitue l'intégrité de l'objet. Dans cette expérience, les verbes sont donnés hors contexte (par ordre alphabétique) : on subit nécessairement les conséquences de cette imprécision, par exemple avec les verbes jeter et secouer qui se trouvent dans la sous-classe 256 alors qu'on aurait pu les attendre dans la sousclasse 271.

Les verbes de la classe 4 se retrouvent dans la classe 293 qui est trop générale pour pouvoir toujours être représentée par la notion de changement de valeur de l'attribut d'un objet. Les verbes appuyer, toucher, tacher, peindre, couvrir, entourer et envelopper représentent une action sur la frontière d'un objet, les verbes agiter et remuer une action sur l'état interne d'un objet, les verbes de la sous-classe 288 une action (de 
nature non spécifiée) sur un objet. Les verbes de la sous-classe 262 appartiennent au champ défini précédemment comme celui de l'assemblage. Ce qui caractérise finalement cette classe est la présence de la primitive FAIRE appliquée sur un objet.

Deux inverses du point de vue des schèmes, assemblage et désassemblage, appartiennent à des champs différents (contrairement à la classe de possession qui forme un tout quel que soit le sens du transfert). Ce qui prime donc pour la caractérisation de ces champs sémantiques est plutôt la notion de destruction de l'intégrité d'un objet ainsi que celle d'action sur un objet.

La classe 290 reprend les verbes de la classe précédemment dénommée activité, regroupés dans la sous-classe 270 ; elle est enrichie des verbes de la sous-classe 284 . Pour les verbes abandonner et laisser, il y a contrôle mais sur le fait qu'on laisse faire. Pour les autres verbes de cette classe, il y a contrôle avec une visée négative et téléonomique. Les verbes de la sous-classe 284 sont donc liés à la causalité. Les verbes de la sous-classe 270 représentent des activités avec leurs modalités d'action. Ils permettent de caractériser un processus.

La classe 300 reprend les verbes des classes mouvement avec contrôle interne et mouvement avec contrôle externe. Les schèmes de ces deux classes étaient très proches, ce qui explique qu'on les trouve regroupés dans cette seconde analyse. La classe 300 caractérise donc les verbes de mouvement.

En prenant comme critère les fréquences moyennes d'association, on se rend compte que certains verbes se retrouvent dans une classe particulière parce qu'ils ne peuvent pas aller ailleurs (c'est notamment le cas lorsque le critère a une valeur proche de 1). Les verbes les plus représentatifs ont une fréquence moyenne d'association supérieure à 2 .

Les deux analyses font apparaître une organisation des champs différente mais sans incompatibilité. Les champs dégagés en première analyse se retrouvent, mais regroupés avec d'autres pour recevoir une interprétation plus générale, ou parfois éclatés pour faire apparaître un autre type d'organisation générale (assemblage / désassemblage).

Les champs sémantiques sont caractérisés par une notion. Par exemple pour le champ de transfert c'est la notion de possession " aposs " qui pourra engendrer des schèmes divers en se composant avec des primitives (IDENT, CHANG). Un champ sémantique définit une ressemblance, il n'y a donc pas de schème associé. Les champs seront très souvent associés à une primitive ; on va donc retrouver certaines des primitives de la GA\&C.

\subsection{Interprétation de l'expérience 2, réplique de l'expérience 1}

Bien que les classes dégagées par les expériences ne soient pas exactement identiques, principalement dans leur organisation les unes par rapport aux autres, nous retrouvons quelques grands groupes de signification :

- un groupe organisé autour de la primitive de possession, comprenant les verbes exprimant soit un transfert de possession, soit une conservation de possession ;

- un groupe organisé autour de la primitive FAIRE qui s'applique sur un objet ; 
- un groupe organisé autour de la notion de processus, les verbes de cette classe caractérisant différents aspect d'un processus ;

- un groupe organisé autour de la notion d'agression, soit rapproché des activités de base (l'agression serait alors une des activités de base), soit rapproché de verbes exprimant la perte d'intégrité d'un objet ;

- un groupe organisé autour de la notion d'activités de base ;

- un groupe organisé autour de la notion de dissociation d'un tout en ses parties ;

- enfin un groupe organisé autour de la primitive de mouvement.

Mis à part les classes des verbes d'agression qui ont une structure complexe et des verbes d'activités de base qui eux par contre sont primitifs et non descriptibles par des schèmes, les autres classes peuvent être représentées par des primitives de la GA\&C : le relateur de possession aposs, la primitive FAIRE, la notion de processus ou de lieu d'activité, la notion d'ingrédience (qui est ici niée) et enfin la primitive MOUVT.

\subsection{Les relations entre un champ sémantique et un schème}

Chaque item verbal définit un réseau de significations que nous représentons par des schèmes. Dans une tâche de classement, rapprocher plusieurs verbes consiste à extraire du réseau de chacun d'eux un schème particulier tel que les schèmes dégagés aient des propriétés communes. Ces propriétés communes à plusieurs schèmes peuvent éventuellement être représentées par une notion abstraite qui caractériserait alors un champ sémantico-cognitif.

Certains verbes ont changé de classe d'une expérience à l'autre : le fait de ranger un item verbal dans une classe plutôt qu'une autre suppose de la part du sujet le choix d'une signification particulière du réseau. Ceci explique que certains verbes soient associés à une certaine classe dans une expérience, et à une autre classe dans l'autre expérience.

Comme un verbe admet plusieurs significations, un verbe peut appartenir à plusieurs champs sémantico-cognitifs. Par exemple le verbe achever, dans la première expérience, est rapproché des verbes détruire et tuer dans la classe représentant les verbes d'agression, alors qu'il est rapproché du verbe réussir de la classe des verbes caractérisant un processus dans la deuxième expérience. Ces deux rapprochements sont autant justifiés l'un que l'autre, ils manifestent simplement la polysémie verbale. Dans la première expérience, achever est pris dans le sens de Paul achève Pierre avec une matraque, dans la seconde expérience dans le sens de Paul achève la rédaction de son mémoire. Mais, et c'est ce qui est intéressant ici, nous constatons que, d'une manière globale, les classes sont conservées. Les variantes sont plutôt d'ordre local.

\section{Conclusion}

Les résultats des différentes analyses que nous avons faites sur les données des deux expériences de catégorisation des verbes d'action sont relativement cohérents. Si l'on en fait la synthèse, on retiendra que six groupes sémantiques se dégagent :

- les verbes exprimant le mouvement ou le déplacement d'une entité

- les verbes exprimant la possession ou le transfert de possession 
- les verbes exprimant soit la construction d'une nouvelle entité par assemblage soit la modification d'une propriété d'une entité

- les verbes exprimant une atteinte à l'intégrité physique d'une entité (attaque, destruction)

- les verbes exprimant les activités de base du vivant

- les verbes exprimant les processus qui décrivent le cours de l'action

À tous ces groupes peuvent être associés des primitives ou des notions qui décrivent les significations partagées par l'ensemble des verbes du groupe. On peut donc caractériser de façon précise la structure sémantique de chacun de ces groupes. Il y a une convergence manifeste entre les résultats d'une analyse psychologique des significations d'actions telles qu'on peut les appréhender à partir de tâches de catégorisation et les résultats d'une analyse linguistique fondée sur une approche formelle de l'analyse des significations.

Si l'on examine le contenu sémantique des six groupes de verbes qui apparaissent dans l'analyse, on constate que ces groupes expriment bien les grands mobiles de l'action humaine et les grandes catégories d'objectifs ultimes que l'on peut assigner à cette activité. Ainsi, ces groupes peuvent être considérés comme une première approximation des champs sémantico-cognitifs.

Il reste beaucoup à faire pour délimiter et caractériser ces champs. Il est vraisemblable que certains champs n'aient pu être identifiés en utilisant les 172 verbes que nous avons choisis à partir du critère de fréquence. Il est possible en effet qu'il existe d'autres communautés de signification qui soient exprimées par des verbes plus rares : ainsi les verbes exprimant des transformations techniques ne sont pas des verbes fréquents en regard des critères utilisés par les dictionnaires de fréquence. De plus, des incertitudes demeurent quant au fait de savoir si des groupes proches constituent ou non des champs sématiques distincts ; c'est le cas des verbes de mouvement et des verbes de déplacement, c'est le cas aussi des verbes qui expriment l'agression et de ceux qui expriment la destruction d'objets.

Un autre travail reste à faire, qui est de décrire l'organisation sémantique à l'intérieur de chaque champ d'une façon qui soit compatible d'une part avec les données psychologiques concernant l'appréhension des significations par les sujets et d'autre part les analyses linguistiques qui essaient de caractériser de façon formelle les significations lexicales des verbes dans leurs différents environnements actanciels.

Ces premiers résultats nous semblent montrer qu'il est nécessaire de distinguer trois niveaux dans l'analyse des significations. Les primitives ont le statut d'éléments qui entrent dans la composition des schèmes sémantico-cognitifs et dans la caractérisation des champs sémantico-cognitifs, de sorte qu'elles ne prennent leur sens que dans le cadre de structures plus larges. Deux types de structures plus complexes que les primitives doivent être distinguées : ce sont d'une part les schèmes sémantico-cognitifs qui permettent de décrire et d'expliquer les différentes acceptions d'un verbe et d'autre part les champs sémantico-cognitifs qui permettent de décrire et d'expliquer les communautés de signification portées par des verbes dont les acceptions sont décrites par des schèmes différents mais qui partagent néanmoins une parenté de signification très importante, qui est bien mise en évidence dans les tâches de catégorisation. 


\section{Bibliographie}

Aвrahaм, M. (1995). Schèmes sémantico-cognitifs des verbes (verbes de mouvement), EHESS Paris.

Barthélémy J. P., Guenoche A. (1988). Les arbres et les représentations de proximités. Paris : Masson.

Juilland A., Brodin D., Davidovitch C. (1970). Frequency dictionnary of french words. The Hague : Paris, Mouton.

Meunier J. M. (1992) Relations et organisation interconceptuelles des verbes de déplacement et de mouvement. Mémoire de DEA, Université de Paris 8.

Miller, G. A. (1969) A psychological method to investigate verbal concepts. Journal of Mathematical Psychology, 6, 169-171.

TetsCH D. (1991) La Sémantique des actions : une étude à partir d'actions mises hors et en contexte d'objets. Mémoire de DEA, Université de Paris 8.

\section{Annexe 1 : liste des verbes}

Les verbes marqués d'un astérique sont ceux qui ont été enlevés pour la seconde analyse, parce qu'étant associés à plusieurs classes.

Abandonner*, accompagner, accoucher*, acheter, achever*, acquérir, agiter, agrandir, ajouter*, aller, allumer*, amener*, appliquer*, apporter*, approcher, appuyer, arracher, arrêter, arriver*, arroser*, assembler*, attacher*, attaquer, atteindre, augmenter, avaneer, baisser, battre, boire, bouger, briser, casser, cesser, changer*, chanter, chasser, cogner, combattre, commencer, conduire, conserver, continuer, couper, courir*, couvrir*, créer, crier, danser, déformer*, déplacer*, descendre, détacher*, détruire, disperser, disposer*, donner, écarter*, effacer, éloigner*, embrasser, emmener*, empêcher, empêcher de, employer, emporter*, endormir, enfoncer*, enlever*, entourer, entraîner, entrer, envelopper*, envoyer*, ́́pouser*, essayer, éteindre* , éviter, exercer, faire*, fermer*, finir, fixer*, fournir*, frapper, fuir, garder, glisser*, interrompre, jeter*, jouer, laisser, lancer*, lever*, livrer, lutter, maintenir*, manger, marcher*, marier*, mener*, mettre*, monter, murmurer, nourrir, obtenir, offrir, ouvrir, parcourir, partager, partir, parvenir*. passer, payer, peindre*, pénétrer, perdre, placer*, pleurer, porter, poser, poursuivre*, prendre*, préparer, prêter, produire, promener, quitter, rapprocher, rassembler*, réaliser, recevoir, reculer, rejoindre, remplir, remuer, rencontrer, rendre, réparer, respirer, retirer*, réunir, réussir, réveiller, rompre, rouler, s'asseoir*, s'échapper, saisir*, sauter*, se coucher*, se mettre à, secouer, séparer, serrer*, sortir, souffler, soulever*, supporter*, tâcher, tacher, tenir*, terminer, tirer*, toucher, tourner, traîner*, travailler, traverser, trembler, tuer, vendre, venir.

\section{Annexe 2 : \\ Les champs sémantiques}

\section{Champ I}

Accompagner, aller, approcher, arriver, atteindre, avancer, bouger, conduire, courir, danser, déplacer, descendre, entraîner, entrer, fuir, glisser, marcher, mener, monter, parcourir, partir, parvenir, passer, pénétrer, poursuivre, promener, quitter, reculer, rejoindre, rencontrer, rouler, s'asseoir, s'échapper, sauter, se coucher, sortir, tourner, traîner, traverser, venir.

Champ 2

Amener, appliquer, apporter, conduire, déplacer, descendre, détacher, éloigner, emmener, emporter, envoyer, glisser, jeter, lancer, lever, mettre, monter, placer, porter, poser, prendre, rapprocher, saisir, soulever, supporter, tirer, tourner, traîner.

Champ 3

abandonner, acheter, acquérir, conserver, donner, fournir, garder, livrer, obtenir, offrir, partager, payer, perdre, prendre, prêter, recevoir, rendre, vendre.

Champ 4

Agiter, agrandir, allumer, arroser, augmenter, baisser, casser, changer, couper, couvrir, déformer, écarter, entourer, envelopper, éteindre, fermer, lever, ouvrir, peindre, remplir, remuer, réparer, rompre, serrer, tacher. 


\section{Champ 5}

Ajouter, amener, appliquer, appuyer, assembler, attacher, changer, couvrir, détacher, disperser, disposer, écarter, enfoncer, enlever, entourer, envelopper, fixer, laisser, maintenir, mettre, placer, rapprocher, rassembler, réparer, retirer, réunir, séparer, serrer,

Champ 6

Achever, arracher, briser, casser, chasser, couper, descendre, détruire, effacer, enlever, jeter, partager, prendre, rompre, tuer.

Champ 7

Achever, agiter, arracher, attaquer, battre, chasser, cogner, combattre, crier, détruire, empêcher, entraîner, frapper, fuir, jeter, livrer, lutter, s'échapper, secouer, tuer.

Champ 8

Abandonner, accoucher.. achever, arrêter, arriver, atteindre, cesser, commencer, continuer, créer, empêcher, empêcher de, employer, essayer, éviter, exercer, faire, finir, fournir, fuir, interrompre, laisser, mener, obtenir, parvenir, poursuivre, préparer, produire, réaliser, réussir, se mettre à, tâcher, terminer, travailler. 